\title{
IJCARS-MICCAI 2018 special issue
}

\author{
Gabor Fichtinger $^{1}$ - Julia A. Schnabel ${ }^{2} \cdot$ Christos Davatzikos $^{3}$ - Alejandro F. Frangi ${ }^{4} \cdot$ Carlos Alberola-López $^{5}$
}

Published online: 14 September 2019

(c) CARS 2019

It is our pleasure to introduce this special issue of IJCARS, selected amongst the best papers presented at the 21st Medical Image Computing and Computer-Assisted Intervention (MICCAI 2018) Conference, held in Granada, Spain.

For MICCAI 2018, a total of 1335 papers were submitted, of which 1066 manuscripts were sent for peer review by 3 experts each after editorial triage. Following a double-blind review process, 372 papers $(34.9 \%)$ were accepted for presentation at the conference in either podium or poster format.

Papers that received the highest review scores and specifically pertained to the general topic of computer-assisted intervention were invited to this special issue of IJCARS. Authors had to significantly extend the contents of their MICCAI conference papers, undergo a full new peer review process, and meet the Journal's rigorous standards of scientific novelty and quality. We invited 22 papers, 14 of which are included in this volume.

\footnotetext{
Gabor Fichtinger

gabor.fichtinger@queensu.ca

Julia A. Schnabel

julia.schnabel@kcl.ac.uk

Christos Davatzikos

christos.davatzikos@pennmedicine.upenn.edu

Alejandro F. Frangi

a.frangi@leeds.ac.uk

Carlos Alberola-López

caralb@tel.uva.es

1 School of Computing, Queen's University, 25 Union St., Kingston, ON K7L 2N8, Canada

2 School of Biomedical Engineering and Imaging Sciences, King's College London, Becket House, 1 Lambeth Palace Road, London SE1 7EU, UK

3 Departments of Radiology, and Electrical and Systems Engineering, University of Pennsylvania, 3600 Market Street, Philadelphia, PA 19104, USA

4 School of Computing and School of Medicine, University of Leeds, Woodhouse Lane, Leeds LS2 9JT, UK

5 ETSI Telecomunicación, Universidad de Valladolid, Paseo de Belén 15, 47011 Valladolid, Spain
}

The wide spectrum of papers reflects recent trends in computer-assisted interventions, including an increasing interest in machine learning approaches to problems like segmentation, registration, tracking, image simulation and classification with a focus on image-guided interventions, and intuitive innovative solutions for visualization and augmented reality surgical navigation.

This special issue aims to provide a representative collage of the diverse topics covered in computer-assisted interventions at MICCAI 2018. We would like to thank the authors, reviewers, and the Editorial Office of IJCARS for their efforts to bring forth this special issue we hope you will enjoy reading.

Publisher's Note Springer Nature remains neutral with regard to jurisdictional claims in published maps and institutional affiliations. 